\title{
Hipertensión arterial en pacientes de edad avanzada: una revisión estructurada
}

\author{
Edwin J. Osorio-Bedoya ${ }^{\mathrm{a}, \mathrm{c}, \mathrm{d}, *}$ y Pedro Amariles ${ }^{\mathrm{a}, \mathrm{b}, \mathrm{c}}$
}

a Departamento de Farmacia, Facultad de Ciencias Farmacéuticas y Alimentarias, Universidad de Antioquia, Medellín, Colombia

b Grupo de Investigación en Atención Farmacéutica de la Universidad de Granada, España

c Grupo de Investigación Promoción y Prevención Farmacéutica, Facultad de Ciencias Farmacéuticas y Alimentarias, Universidad de Antioquia, Medellín, Colombia

d Grupo de Investigación Tecnología en Regencia de Farmacia. Facultad de Ciencias Farmacéuticas y Alimentarias, Universidad de Antioquia, Medellín, Colombia

Recibido el 31 de octubre de 2016; aceptado el 3 de octubre de 2017

Disponible en Internet el 17 de abril de 2018

\section{PALABRAS CLAVE \\ Hipertensión; Factores de riesgo cardiovascular; Tratamiento; Presión arterial; Anciano}

\begin{abstract}
Resumen
Introducción: La hipertensión arterial es frecuente y sigue siendo un factor de riesgo importante en pacientes de edad avanzada.

Objetivo: Identificar las características más importantes y las recomendaciones actuales en el tratamiento de la hipertensión arterial en pacientes de edad avanzada.

Método: Se realizó una revisión estructurada en PubMed/Medline de trabajos relacionados con hipertensión en edad avanzada entre los años 2005 y 2015. Para ello, se utilizó la siguiente estrategia de búsqueda libre: "(hypertension) AND (drug therapy) AND (elderly OR older person)", que se encontraran en el título o el resumen.

Resultados: Se seleccionaron 83 artículos de la revisión estructurada relacionados con el tema de hipertensión en edad avanzada; se identificaron los aspectos acerca del tratamiento, la valoración de la necesidad, los objetivos terapéuticos y las características relacionadas con la seguridad del tratamiento farmacológico.

Conclusiones: Se identificaron las características más importantes del tratamiento de la hipertensión en edad avanzada y se establecieron recomendaciones de utilidad para el tratamiento de estos pacientes.

(c) 2018 Sociedad Colombiana de Cardiología y Cirugía Cardiovascular. Publicado por Elsevier España, S.L.U. Este es un artículo Open Access bajo la licencia CC BY-NC-ND (http:// creativecommons.org/licenses/by-nc-nd/4.0/).
\end{abstract}

\footnotetext{
* Autor para correspondencia.

Correos electrónicos: jair.osorio@udea.edu.co, edwinjair@hotmail.com (E.J. Osorio-Bedoya).
} 


\section{KEYWORDS}

Hypertension;

Cardiovascular risk

factors;

Treatment;

Blood pressure;

Elderly

\section{Arterial hypertension in patients of advanced age. A structured review}

\begin{abstract}
Introduction: Hypertension is common, and continues to be a significant risk factor in patients of advanced age.

Objective: To identify the most important features and the current recommendations in the treatment of arterial hypertension in patients of advanced age.

Method: A structured review was performed using works found in PubMed/Medline related to hypertension in advanced age between the years 2005 and 2015. The following free search strategy was used for this: "(hypertension) AND (drug therapy) AND (elderly OR older person)", which would be found in the title or abstract.

Results: A total of 83 articles associated with the subject of hypertension in advanced age were selected for the structured review. Aspects on the treatment were identified, as well as the assessment of the need, the therapeutic aims, as well as the characteristics associated with the safety of the pharmacological treatment.

Conclusions: The most important features of the treatment of hypertension in advanced age are identified, and useful recommendations were established for the treatment of these patients. (C) 2018 Sociedad Colombiana de Cardiología y Cirugía Cardiovascular. Published by Elsevier España, S.L.U. This is an open access article under the CC BY-NC-ND license (http:// creativecommons.org/licenses/by-nc-nd/4.0/).
\end{abstract}

\section{Introducción}

La hipertensión arterial (HTA) es una enfermedad crónica caracterizada por el incremento continuo de las cifras de la presión sanguínea en las arterias, y es un factor de riesgo para eventos cardiovasculares, incluidos los accidentes cerebrovasculares isquémico y hemorrágico. En este sentido, Margaret Chan, Directora General de la Organización Mundial de la Salud (OMS), declaró que la hipertensión afecta a mil millones de personas en el mundo, y puede provocar infartos de miocardio y accidentes cerebrovasculares. Los investigadores calculan que la hipertensión es la causa por la que mueren anualmente nueve millones de personas ${ }^{1}$. Por su parte, el control adecuado de las cifras de presión arterial, a través de medidas como modificaciones en los estilos y hábitos de vida, al igual que del tratamiento farmacológico antihipertensivo, reduce el ataque cerebrovascular en hombres y mujeres de edad avanzada.

Existen numerosos factores relacionados con el comportamiento que pueden contribuir a que se presente la hipertensión, entre ellos: el consumo de alimentos que contienen exceso de sodio y de grasas saturadas, la ingesta insuficiente de frutas y verduras, el alto consumo de bebidas alcohólicas, el sedentarismo, la falta de ejercicio físico y el estrés. Las condiciones de vida y trabajo de las personas influyen notoriamente en estos factores de riesgo conductuales. Además, existen algunos factores metabólicos que aumentan el riesgo de enfermedad cardiovascular, ataque cerebrovascular, insuficiencia renal y otras complicaciones de la hipertensión, como los son la diabetes, la hipercolesterolemia y el sobrepeso o la obesidad. El consumo de cigarrillo y la hipertensión interactúan para aumentar aún más la probabilidad de padecer enfermedad cardiovascular (ECV).

Referente al tratamiento de la HTA en pacientes de edad avanzada, las recomendaciones de las sociedades científicas internacionales difieren en umbrales y metas para el tratamiento farmacológico antihipertensivo ${ }^{2}$, debido a que la evidencia es limitada y a que no existe un consenso en el concepto de "pacientes de edad avanzada".

La población mayor de 75 años crece de manera vertiginosa, principalmente en los países desarrollados; por tanto, se necesitan cada vez más directrices que permitan abordar a estos "pacientes de edad muy avanzada". En este sentido, los objetivos terapéuticos en estos grupos, deben pasar más que de la prolongación de la vida, a un enfoque en la calidad de vida y al mantenimiento de sus funciones ${ }^{3,4}$.

La terapia antihipertensiva parece ser clínicamente beneficiosa en pacientes de edad avanzada, ya que disminuye la morbi-mortalidad cardiovascular. Al respecto, la combinación de medicamentos, la adherencia terapéutica y la identificación de la dosis más baja eficaz, se han relacionado con mayor efectividad y seguridad del tratamiento antihipertensivo ${ }^{2}$. Sin embargo, la información relacionada con las características clínicas y el tratamiento adecuado de la HTA en pacientes de edad avanzada se considera limitada y fraccionada.

\section{Objetivo}

Identificar y sistematizar información relacionada con las características clínicas de la hipertensión en edad avanzada, los aspectos relacionados con el tratamiento, la valoración de la necesidad, efectividad y seguridad de los medicamentos, y los aspectos prácticos y recomendaciones en el tratamiento de la hipertensión arterial en pacientes de edad avanzada.

\section{Método}

Se efectuó una revisión estructurada en PubMed/Medline de trabajos relacionados con hipertensión en edad avanzada. Para ello se utilizó la siguiente estrategia de búsqueda libre: "(hypertension) AND (drug therapy) AND (elderly OR older person)', que se encontrara en el título o el resumen. 


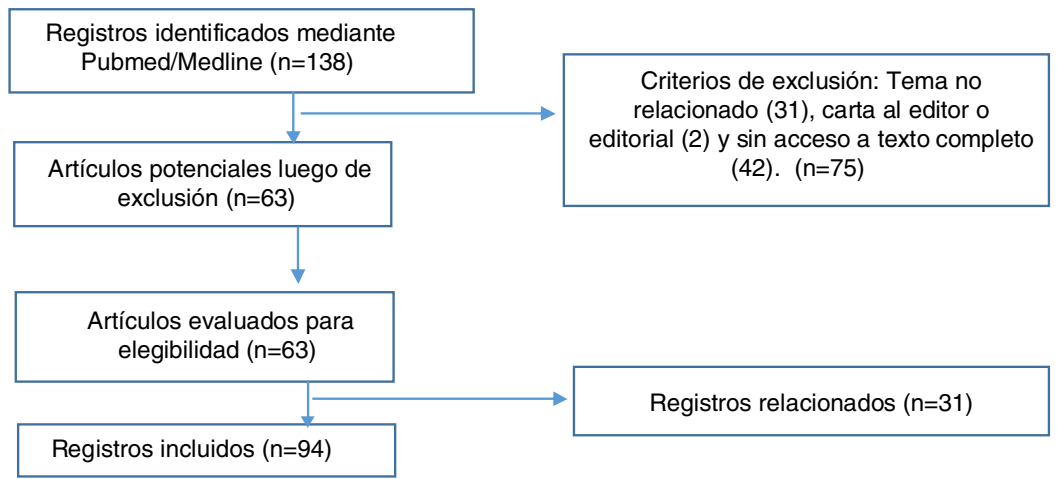

Figura 1 Flujo de la búsqueda, identificación y selección de artículos incluidos en la revisión.

Se incluyeron artículos en inglés o español, hechos en humanos y publicados entre enero de 2005 y agosto de 2015, y con acceso a texto completo. Se revisaron y se incluyeron aquellos que presentaban resultados relacionados directamente con el objetivo de la revisión. Por su parte, se excluyeron artículos no relacionados con el tema a investigar y cartas al editor.

Los artículos incluidos fueron leídos y analizados para identificar las características más importantes de la hipertensión en edad avanzada. En este sentido, la información se agrupó en las siguientes categorías:

1) Características clínicas de la hipertensión en edad avanzada.

2) Aspectos relacionados con el tratamiento.

3) Valoración de la necesidad, efectividad y seguridad de los medicamentos.

4) Aspectos prácticos y recomendaciones en el tratamiento de la hipertensión arterial.

\section{Resultados}

La figura 1 indica el flujo de búsqueda, identificación y selección de los trabajos incluidos en la revisión.

\section{Características clínicas de la hipertensión en edad avanzada}

El grupo poblacional de 60 años o más se caracteriza por su aumento progresivo y por una prevalencia elevada de HTA. Por ello, en este grupo, la valoración de la presión arterial (PA) es muy importante, ampliamente aplicable y necesaria para un adecuado control de la $\mathrm{HTA}^{5}$. Además, es destacable que la hipertensión sistólica aislada es la que aparece con mayor frecuencia en este grupo poblacional ${ }^{6}$. En este contexto, el aumento de la presión arterial sistólica se asocia con resultados adversos, incluyendo ataque cerebrovascular, enfermedad cardiovascular y muerte. En este grupo de pacientes la hipertensión puede atribuirse a numerosos cambios estructurales y funcionales, como por ejemplo, la vasculatura que se desarrolla con el aumento de la edad ${ }^{7}$.

La hipertensión arterial es un trastorno dependiente de la edad, por tanto se debería disponer de recomendaciones específicas para su tratamiento en los pacientes de edad avanzada. Las modificaciones de estilo de vida y el uso de productos naturales y terapias hormonales, son útiles para las etapas iniciales y sirven para apoyar el tratamiento farmacológico, pero se requiere evidencia con ensayos clínicos en esta población. En general, los agentes antihipertensivos pueden reducir la presión arterial en las personas de edad avanzada, por tanto, la terapia debe basarse en sus posibles efectos secundarios e interacciones medicamentosas ${ }^{8}$.

En pacientes de edad avanzada, el deterioro de la función de los vasos sanguíneos puede conducir al padecimiento de la ECV. Los mecanismos subyacentes al endotelio, al músculo liso vascular y la disfunción de la matriz extracelular, relacionados con la edad, son causados por el estrés oxidativo, la reducción del óxido nítrico o su aumentada eliminación, el desequilibrio de la relación vasoconstrictor/vasodilatador, el grado bajo del ambiente pro-inflamatorio, la angiogénesis alterada y la senescencia celular endotelial. En resumen, el proceso de envejecimiento en el músculo liso vascular se caracteriza por el potencial de replicación alterada, el cambio en el fenotipo celular y la capacidad de respuesta a los mediadores de la contracción y la relajación, y por último, por los cambios en las funciones de señalización intracelular ${ }^{9}$. A continuación se describirán las principales características clínicas que se presentan en mayor proporción en los pacientes de edad avanzada.

\section{Hipertensión arterial sistólica}

Es un importante problema de salud en los pacientes de edad avanzada. Se caracteriza por presión arterial sistólica $\geq 140 \mathrm{~mm} \mathrm{Hg}$, presión arterial diastólica < $90 \mathrm{~mm} \mathrm{Hg} \mathrm{y,} \mathrm{en}$ consecuencia, presión de pulso por encima de los $50 \mathrm{~mm} \mathrm{Hg}$. Este problema de salud afecta a más del $50 \%$ de los pacientes mayores de 60 años ${ }^{10}$, y es una causa importante de ataque cerebrovascular, así como de enfermedad cardiovascular y renal $^{11}$. La presión de pulso elevada es una consecuencia y un indicador de las arterias rígidas en los pacientes con hipertensión arterial sistólica, y se ha demostrado que es un predictor de la disminución de la función endotelial, apoplejía, enfermedad cardiovascular y mortalidad ${ }^{12,13}$.

La rigidez arterial aumentada es el fenotipo vascular de la hipertensión sistólica, especialmente de las grandes arterias. Este es un fenómeno propio en la edad avanzada y se debe principalmente a la fractura de las laminillas de elastina en la pared de la aorta, de manera que, los tramos de la 
pared tienen mayor distensión de las fibras de colágeno, lo que causa mayor rigidez ${ }^{14}$. La presión arterial sistólica elevada, en comparación con la diastólica, se asocia más con la morbilidad y mortalidad cardiovascular ${ }^{15-17}$.

El tratamiento de la hipertensión sistólica en edad avanzada, debe basarse principalmente en medidas no farmacológicas, y sólo en los casos en que estas medidas no funcionen, se recurrirá al tratamiento farmacológico. La hipertensión sistólica es difícil de tratar, posiblemente, debido a que el problema de fondo son los cambios estructurales en las arterias de conducción. Por ello, más del $60 \%$ de los pacientes con hipertensión sistólica tienen mal control de la presión arterial y no cumplen con el objetivo clínico de $140 / 90 \mathrm{~mm} \mathrm{Hg}^{18}$ o presión de pulso de $50 \mathrm{~mm} \mathrm{Hg}$. La reducción de la presión arterial se puede lograr con medidas comportamentales y de estilo de vida, como el yoga y la meditación, y se cree que la respiración lenta controlada, puede ser el factor común responsable de la mejoría ${ }^{19,20}$.

El estudio Hypertension in the Very Elderly Trial ${ }^{21}$ (HYVET, su sigla en inglés), es un ensayo aleatorizado, doble ciego, controlado realizado en 195 centros en 13 países de Europa occidental y oriental, China, Australia y África del Norte, que proporciona datos de medicina basada en la evidencia en pacientes mayores de 80 años, y demuestra que lograr disminuir las cifras de presión arterial en estos pacientes, a un nivel de $150 / 80 \mathrm{~mm} \mathrm{Hg}$ es muy beneficioso.

Más recientemente, el octavo Comité Nacional Conjunto (JNC-8), recomienda que en los adultos mayores de 60 años, puede considerarse como meta terapéutica, lograr y mantener cifras de presión sistólica hasta menores a los $150 \mathrm{~mm} \mathrm{Hg}$. El tratamiento antihipertensivo debe adaptarse en este grupo de pacientes debido a condiciones comórbidas, como enfermedad isquémica del corazón, insuficiencia cardíaca, fibrilación auricular, insuficiencia renal y diabetes. Por ejemplo, los inhibidores de la enzima convertidora de angiotensina (IECA) y los bloqueadores de los receptores de la angiotensina II (ARA-II), deben considerarse en combinación con diuréticos, o con un calcioantagonista. Los betabloqueadores parecen ser menos eficaces para la protección de enfermedades cardiovasculares en comparación con otras clases de antihipertensivos, tales como diuréticos, dihidropiridinas, IECA o ARA-II ${ }^{15}$.

Por su parte, en un estudio en pacientes con HTA sistólica, en el que se empleó el régimen basado en la clortalidona, se demostró una reducción en la incidencia de ictus del $36 \%$, de infarto de miocardio (IM) del $27 \%$, de insuficiencia cardiaca (IC) del $54 \%$ y de morbilidad cardiovascular general en un $32 \%{ }^{17}$.

Es importante mencionar que se requiere un mayor esfuerzo para reducir la inercia terapéutica y aumentar la adherencia a fin de optimizar el control de la presión arterial en los pacientes de edad avanzada con hipertensión sistólica ${ }^{15}$.

\section{Hipertensión y enfermedad coronaria}

La hipertensión arterial es un factor de riesgo independiente importante para el desarrollo de enfermedad coronaria, ataque cerebrovascular e insuficiencia renal ${ }^{22}$. La enfermedad coronaria, conocida también como enfermedad de las arterias coronarias, es una afección en la que una placa de ateroma se deposita en las arterias coronarias y causa aterosclerosis, que se puede manifestar como angina estable o como síndrome coronario agudo (angina inestable o infarto agudo de miocardio).

La elección óptima de agentes antihipertensivos sigue siendo controvertida y sólo se dispone de respuestas parciales a las preguntas importantes en el tratamiento de la hipertensión para la prevención y el manejo de la cardiopatía isquémica ${ }^{22}$. Además, la hipertensión afecta a dos tercios de los adultos mayores y aún es un factor de riesgo potente para enfermedad arterial coronaria, insuficiencia cardíaca crónica, fibrilación auricular e ictus en este grupo de edad ${ }^{23}$. Existe, no obstante, claridad en que el uso de antihipertensivos en personas mayores de 60 años, se asocia a disminución del riesgo de enfermedad coronaria y enfermedad cerebrovascular. De forma similar, el tratamiento antihipertensivo se relaciona con disminución del riesgo de enfermedad coronaria y enfermedad cerebrovascular en mujeres de cualquier edad y origen racial. En tal sentido, el uso de antihipertensivos en monoterapia en pacientes con tensión arterial media menor a 160/90, o terapia combinada en pacientes con tensión arterial media mayor, se asocia con disminución del riesgo de enfermedad coronaria y enfermedad cerebrovascular. En la prevención de la enfermedad cerebrovascular, a excepción de un probable efecto mayor de los calcioantagonistas, no existen diferencias en el beneficio obtenido con los diferentes tipos de antihipertensivos ${ }^{24}$.

Por otro lado, en pacientes de edad avanzada con enfermedad coronaria, es frecuente el síndrome de fragilidad, e implica una disminución fisiológica progresiva en la reserva y la función a través de múltiples sistemas fisiológicos. La presencia de fragilidad tiene un impacto negativo en la calidad de vida temprana y requiere, por tanto, de estrategias terapéuticas y colaboración del personal de enfermería para su tratamiento ${ }^{25}$.

\section{Aspectos relacionados con el tratamiento}

En diciembre de 2013, el JNC-8 presentó sus nuevas directrices sobre el manejo de la hipertensión en adultos ${ }^{26}$. En comparación con el JNC-7, los cambios principales incluyen las metas en la presión arterial y los umbrales para la iniciación del tratamiento de los pacientes de edad avanzada y en menores de 60 años con diabetes o enfermedad renal ${ }^{27}$. Por su parte, en 2013, la Sociedad Europea de Hipertensión (ESH) y la Sociedad Europea de Cardiología (ESC) publicaron directrices conjuntas para el tratamiento de la hipertensión ${ }^{28}$. De forma similar, la Sociedad de Hipertensión de Suráfrica (SAHS), publicó en 2014 una nueva directriz de tratamiento y prevención de la $\mathrm{ECV}^{29}$. Más recientemente, los miembros del Comité Ejecutivo de la Sociedad Taiwanesa de Cardiología (TSOC) y la Sociedad de Hipertensión de Taiwán (THS), con el objetivo de revisar la información sobre el manejo de la hipertensión, publicaron una guía actualizada del tratamiento de la hipertensión en dicho país. Las directrices TSOC/THS proporcionan la información más actualizada disponible en el manejo de la hipertensión ${ }^{30}$. Más recientemente, el estudio SPRINT ${ }^{31}$ comparó el beneficio del tratamiento del control de la presión arterial sistólica a un objetivo de menos de $120 \mathrm{~mm} \mathrm{Hg}$, frente al tratamiento con un objetivo de menos de $140 \mathrm{~mm} \mathrm{Hg}$, en pacientes de 
edad avanzada con alto riesgo cardiovascular, pero sin diabetes o ictus previo. Para el caso de Colombia, en la actualidad se cuenta con la Guía de práctica clínica de hipertensión arterial primaria, elaborada por el Ministerio de Salud y Protección Social y Colciencias en 2013, la cual pretende desarrollar una serie de recomendaciones basadas en la evidencia, con la mayor validez, claridad y aplicabilidad posible sobre la prevención, el diagnóstico, el tratamiento integral y el seguimiento de pacientes con HTA ${ }^{24}$.

\section{Tratamiento no farmacológico}

La adopción de estilos de vida saludable (plan alimentario adecuado, actividad física regular y mantenimiento del peso corporal ideal) es la piedra angular en el control de la HTA y se asocia con beneficios que van más allá de la reducción de la presión arterial (PA). Una intervención integral del estilo de vida, puede reducir, en muchos casos, la necesidad de medicamentos antihipertensivos en los pacientes de edad avanzada $^{32,33}$.

El JNC-8 concluye que, en general, para todas las personas con hipertensión, un plan alimentario saludable, el control del peso y realizar actividad física de manera regular, tienen el potencial de mejorar el control de la presión arterial y reducir las necesidades de medicamentos. Sin embargo, aunque estas recomendaciones están basadas en la evidencia, no sustituyen el juicio clínico, y las decisiones del manejo deben considerarse cuidadosamente, incorporando las características y circunstancias clínicas de cada paciente ${ }^{26}$.

La guía taiwanesa para el manejo de la hipertensión ${ }^{30}$, adopta la modificación de estilos de vida como parte esencial en la prevención y el control de la hipertensión. En general, se cree que el efecto de la disminución de la presión arterial con la modificación de los estilos de vida, es equivalente a la monoterapia con medicamentos. Si se aplica de manera apropiada la modificación de estilos de vida, puede retrasar el tratamiento farmacológico en pacientes con hipertensión en fase 1 , con la cual se obtiene una reducción en el número y la dosis de medicamentos antihipertensivos. La principal limitación de la modificación de los estilos de vida, radica en la poca continuidad y adherencia. En la tabla 1 se resume la modificación de estilos de vida con el sistema S-ABCDE que comprende: restricción de sodio, disminución del consumo de alcohol, reducción del peso corporal, cese del tabaquismo, adaptación de la dieta y adopción del ejercicio.

Acorde con la Guía práctica clínica de hipertensión para Colombia, existe evidencia de baja a moderada calidad, que sugiere que las estrategias dirigidas a reducir peso, pueden disminuir las cifras de presión arterial. Dicha disminución se ha observado con estrategias combinadas (plan alimentario y ejercicio), o solo con plan alimentario. La población beneficiada es aquella con sobrepeso y obesidad. Además, recomienda implementar planes alimentarios orientados a la disminución de la ingesta de sodio en todos los pacientes con HTA, como recomendación fuerte a favor. Por su parte, no se recomienda incrementar la ingesta de potasio en la dieta o en forma de suplementos dietéticos como medida para prevenir la HTA, como recomendación débil, en contra. También, se indica implementar planes alimentarios dirigidos a la disminución de peso en todos los pacientes con HTA e índice de masa corporal mayor a $25 \mathrm{~kg} / \mathrm{m}^{2}$. Aunque estas recomendaciones no son específicas para pacientes de edad avanzada, son de importante aplicación en todos los grupos de pacientes hipertensos ${ }^{24}$.

\section{Tratamiento farmacológico}

Los medicamentos antihipertensivos, considerados como de primera línea, están enmarcados en los siguientes cinco grupos: diuréticos, betabloqueadores, antagonistas del calcio, IECA y ARA-II. El JNC-8 excluyó como fármacos de primera línea, a los betabloqueadores. De igual manera, la guía clínica de hipertensión de Reino Unido, recientemente, no recomienda el uso de diuréticos y betabloqueantes como tratamiento de primera línea ${ }^{34}$. Sin embargo, las guías europeas de hipertensión ESH/ESC 2013, mantienen estos cinco grupos de antihipertensivos en sus recomendaciones ${ }^{35}$.

Las diferentes directrices señaladas en las distintas guías no son obligatorias, y los miembros de los múltiples grupos de trabajo, coinciden en establecer que el tratamiento de la HTA, debe ser individualizado, acorde con las características clínicas de cada paciente. Por ello, la decisión del médico sigue siendo un factor trascendental en el manejo de la hipertensión. A continuación se detallan algunos aspectos de estos cinco grupos de medicamentos antihipertensivos.

\section{Diuréticos}

Son medicamentos de gran importancia terapéutica en la hipertensión. Reducen eficazmente la PA, y al mismo tiempo pueden disminuir la morbilidad y la mortalidad asociadas con la hipertensión. Además, siguen siendo un componente crucial de la terapia para condiciones de sobrecarga de volumen, como insuficiencia cardíaca (IC), síndrome nefrótico y cirrosis, y para la mejoría de los síntomas de edema ${ }^{17}$.

Los diuréticos exponen varias desventajas con especial relevancia para las personas de edad avanzada. Las alteraciones electrolíticas son los efectos secundarios más reconocidos. La hipopotasemia (hasta $8 \%$ ), no sólo puede precipitar arritmias cardíacas y muerte súbita relacionada, sino también, adinamia por debilidad muscular. La hiponatremia (hasta 17\%) puede contribuir a confusión, delirio y daño cerebral irreversible añadido a la demencia relacionada con la edad.

De acuerdo con el estudio de Wehling ${ }^{36}$, realizado en Alemania, en el tratamiento de la hipertensión, los diuréticos deben ser terceros en la línea de manejo, si el control con los inhibidores del sistema renina-angiotensina-aldosterona (RAA) y los antagonistas del calcio dihidropiridínicos de acción prolongada son insuficientes. Sin embargo, los diuréticos, deben considerarse como una alternativa importante al utilizarse en combinación con los IECA o ARA II, y también con los antagonistas del calcio dihidropiridínicos ${ }^{15}$.

\section{Diuréticos tiazídicos}

Los diuréticos tipo tiazida son agentes de primera línea, útiles en el tratamiento de la hipertensión y han demostrado reducir la mortalidad y morbilidad cardiovascular, adicional a que son fármacos de bajo costo ${ }^{17}$. La hidroclorotiazida 
Tabla 1 Modificación de estilos de vida saludable. S-ABCDE

\begin{tabular}{|c|c|c|c|c|}
\hline Cambio de estilo de vida & Recomendación & $\begin{array}{l}\text { Beneficios esperados en la } \\
\text { reducción de la PAS }\end{array}$ & $\begin{array}{l}\text { Clase de } \\
\text { recomendación }\end{array}$ & $\begin{array}{l}\text { Nivel de } \\
\text { evidencia }\end{array}$ \\
\hline $\begin{array}{l}\text { Sodium restriction (restricción } \\
\text { de sal) }\end{array}$ & 2,0 a 4,0 g/día & $\begin{array}{l}2.5 \mathrm{~mm} \mathrm{Hg} / 1 \mathrm{~g} \text { reducción de } \\
\text { sal }\end{array}$ & I & B \\
\hline $\begin{array}{l}\text { Alcohol limitation (limitación } \\
\text { de alcohol) }\end{array}$ & $\begin{array}{l}\text { Hombres }<30 \mathrm{~g} \text { de } \\
\text { etanol/día Mujeres: }<20 \mathrm{~g} \\
\text { de etanol/día }\end{array}$ & 2 a $4 \mathrm{~mm} \mathrm{Hg}$ & 1 & B \\
\hline $\begin{array}{l}\text { Body weight reduction } \\
\text { (reducción del peso) }\end{array}$ & IMC: 22,5 a 25,0 & $\begin{array}{l}1 \mathrm{~mm} \mathrm{Hg} / \text { por } 1 \mathrm{~kg} \text { de } \\
\text { reducción del IMC }\end{array}$ & 1 & B \\
\hline $\begin{array}{l}\text { Cigarette smoking cessation } \\
\text { (abandono del cigarrillo) }\end{array}$ & Abstinencia completa & Sin efecto independiente & 1 & $\mathrm{C}$ \\
\hline $\begin{array}{l}\text { Diet adaptation } \\
\text { (dieta) }\end{array}$ & $\begin{array}{l}\text { Frutas y verduras ( } 8 \text { a } 10 \\
\text { porciones/día), productos } \\
\text { lácteos bajos en grasa ( } 2 \text { a } 3 \\
\text { porciones/día), reducción } \\
\text { de grasas saturadas y } \\
\text { colesterol }\end{array}$ & 10 a $12 \mathrm{~mm} \mathrm{Hg}$ & 1 & $A$ \\
\hline $\begin{array}{l}\text { Exercise adoption } \\
\quad \text { (ejercicio) }\end{array}$ & $\begin{array}{l}\text { Aeróbico, al menos } 40 \\
\text { minutos/día, y por lo menos } \\
\text { 3-4 días por semana }\end{array}$ & 3 a $7 \mathrm{~mm} \mathrm{Hg}$ & 1 & $A$ \\
\hline
\end{tabular}

Tomada y adaptada de: Chern-En Chiang, Tzung-Dau Wang, Kwo-Chang Ueng, et al. Life style modification for managing hypertension (S-ABCDE). 2015 Guidelines of the Taiwan Society of Cardiology and the Taiwan. Journal of the Chinese Medical Association. 2015;78:1e47.

(HCTZ), la clortalidona y la indapamida se recomiendan como primera línea en el tratamiento de la hipertensión en pacientes de edad avanzada y en general son bien tolerados. Los diuréticos controlan la hipertensión mediante la inhibición de la reabsorción de iones de sodio y cloruro en los túbulos renales. Conducen a una pronta reducción del volumen intravascular y la resistencia vascular periférica, causando una reducción de la $\mathrm{PA}^{37}$.

El ensayo ALLHAT confirmó el efecto equivalente de la clortalidona, con bloqueadores de canales de calcio (BCC) e IECA en la reducción de las enfermedades del corazón; además, la clortalidona fue la mejor estrategia para reducir la $\mathrm{IC}^{30}$. La eficacia de los diuréticos tiazídicos, en la reducción de la IC también se confirmó en un meta-análisis ${ }^{38}$. Cuando la PA no puede ser controlada con IECA o ARA-II, las dosis bajas de diuréticos tiazídicos son un complemento eficaz del tratamiento ${ }^{30}$. Del mismo modo, HYVET proporciona evidencia de que el tratamiento basado en indapamida, con o sin perindopril, en pacientes mayores de 80 años, dirigido a alcanzar una meta de presión arterial de $150 / 80 \mathrm{~mm} \mathrm{Hg}$, es beneficioso y está asociado con disminución de mortalidad e $\mathrm{IC}^{21}$.

\section{Diuréticos de ASA}

Inhiben la reabsorción de sodio y cloro desde la porción ascendente del asa de Henle en el túbulo renal y son útiles sobretodo en situaciones en las que es necesaria una diuresis mayor y más rápida. La furosemida, la bumetanida y la torasemida son los más representativos. Los diuréticos de asa no deben utilizarse como tratamiento de primera línea en la hipertensión, ya que no existen datos de resultados con ellos. Estos diuréticos deben reservarse para las condiciones de sobrecarga de líquidos clínicamente significativa (por ejemplo, la insuficiencia cardíaca y la retención de líquidos) $)^{17}$.

\section{Diuréticos ahorradores de potasio}

Los diuréticos ahorradores de potasio, como la espironolactona, la amilorida y el triamtereno, bloquean los canales de sodio epitelial. Son ampliamente prescritos para la hipertensión como segunda línea en pacientes que toman otros diuréticos (por ejemplo, diuréticos tiazídicos). Sin embargo, hasta el momento no se ha encontrado ningún ensayo que evalúe la eficacia hipotensora de estos medicamentos en monoterapia en pacientes con HTA primaria. Incluso en los ensayos que evalúan la eficacia de dosis bajas de amilorida y triamtereno como un segundo medicamento, no se reduce la $\mathrm{PA}^{17}$. Por tanto, este grupo de medicamentos diuréticos, no deben usarse de manera rutinaria.

\section{Inhibidores de la enzima convertidora de angiotensina} Se destacan en este grupo: enalapril, captopril, ramipril, lisinopril, fosinopril, quinapril, perindopril y benazepril.

Los IECA bloquean la conversión de angiotensina-I a angiotensina-II, que es un vasoconstrictor, lo que lleva a una disminución sistemática de la tensión de los vasos sanguíneos y el volumen sanguíneo, sin afectar la frecuencia cardiaca mediada por estimulación refleja ${ }^{30}$. Los IECA y los ARA-II han demostrado que pueden disminuir la tasa de desarrollo de proteinuria y enfermedad renal diabética. Según la ACC / AHA 2011 (consenso de expertos), las personas de edad avanzada con diabetes, hipertensión y nefropatía debe tratarse inicialmente con IECA o ARA-II, aunque la elección de un antihipertensivo específico, también puede depender de otras comorbilidades asociadas ${ }^{37,39}$. Los IECA han demostrado de forma convincente, reducir las tasas de mortalidad, infarto de miocardio, ataque cerebrovascular y 
revascularización en pacientes con diabetes y antecedentes de enfermedad cardiovascular ${ }^{23}$.

Varios ensayos clínicos hechos en pacientes de edad avanzada con hipertensión, encontraron que los IECA redujeron la presión arterial con la misma eficacia que otros medicamentos, demostrado por menor morbilidad y mortalidad ${ }^{37}$. En el estudio de Pappoe $^{40}$, en el que participaron pacientes diabéticos (edad media 65 años), los criterios de valoración primarios (infarto de miocardio, ictus y muerte cardiovascular) y criterios de valoración secundarios (mortalidad total, insuficiencia cardíaca, y ataque isquémico transitorio), se redujeron significativamente. En general, los beneficios de un IECA o un tratamiento con ARA II son evidentes; sin embargo, se recomienda la monitorización continua de la función renal y de los electrolitos (especialmente potasio). Además, se debe evitar la utilización concomitante de los AINE y de potasio en la dieta. El seguimiento de estas medidas se asocia con una disminución de los problemas de seguridad con esta terapia ${ }^{37}$.

\section{Antagonistas de los receptores de angiotensina-II}

Son la clase más nueva de agentes antihipertensivos utilizados ampliamente en la práctica clínica. Los más representativos son: losartán, olmesartán, telmisartán, candesartán, valsartán e irbesartán. Los ARA-II bloquean la activación los receptores de angiotensina II y son una alternativa cuando los eventos adversos de los IECA no son tolerables. Esto se aplica específicamente a la tos inducida por los IECA. Los ARA-II han demostrado en varios ensayos ser eficaces en la reducción de los niveles de micro-albuminuria y en etapa terminal de enfermedad renal, en pacientes con nefropatía diabética ${ }^{30}$.

Las tasas de eventos adversos son significativamente más bajas que con otras clases de medicamentos antihipertensivos, incluyendo los IECA. Estos factores, dan como resultado un mejor cumplimiento y el aumento de las tasas de adherencia. Estos medicamentos muestran una eficacia similar en la reducción de la presión arterial a otras clases de antihipertensivos y su efecto antihipertensivo se potencia cuando se utilizan de manera concomitante con dosis bajas de diuréticos tiazídicos.

Los ARA-II se eliminan principalmente por vía hepática, pero la mayoría no están sujetos a un amplio metabolismo, y las interacciones con otros medicamentos son poco comunes. Esto es una ventaja en los pacientes de edad avanzada que a menudo reciben múltiples medicamentos, hecho que aumenta el riesgo de interacciones medicamentosas. En general, no se requieren ajustes de dosis en pacientes de edad avanzada, a menos que haya depleción de volumen plasmático ${ }^{41}$.

Su principal desventaja es el costo, pero esto puede ser compensado por su tolerabilidad, ya que tienen menos reacciones adversas y, por tanto, pueden aumentar la adherencia, característica que conduce a un mejor control de la presión arterial y a un menor número de eventos clínicos desfavorables. En general, los ARA-II son bien tolerados y eficaces para disminuir la presión arterial cuando se administran en una sola dosis diaria en pacientes de edad avanzada, y tienen muchos beneficios potenciales en pacientes hipertensos de alto riesgo ${ }^{41}$.
Bloqueadores de los canales de calcio

Inhiben la contracción de las células musculares arteriales coronarias y periféricas, que son considerablemente más dependientes de calcio externo que el corazón y el músculo esquelético. Como resultado, estos medicamentos dilatan las arterias coronarias y periféricas sin poner en peligro el corazón o la contractibilidad del músculo cardiaco. Además, influyen en la función del nódulo sinusal, la conducción aurículo-ventricular y la circulación coronaria ${ }^{30}$. Existen tres subclases: (a) los que bloquean la afluencia de iones de calcio en las células del músculo liso vascular y el tejido miocárdico: fenilalquilaminas (verapamilo), (b) las benzodiacepinas (diltiazem), y (c) las dihidropiridinas (por ejemplo, nifedipino, amlodipino y nitrendipino).

Las directrices del JNC-8, las sociedades americanas e internacionales de hipertensión (ASH/ISH) y la ESH/ESC, incluyen a los bloqueadores de los canales del calcio en la terapia inicial del tratamiento en pacientes hipertensos de edad avanzada. Además, según la guía Surafricana de hipertensión de 2014, los pacientes de raza negra muestran una respuesta más consistente, en comparación con los IECA o los betabloqueadores como monoterapia ${ }^{29}$.

\section{Betabloqueadores}

El mecanismo para la reducción de la presión arterial de los betabloqueadores se basa en la disminución del gasto cardíaco y de la producción de angiotensina II, la inhibición de la liberación de renina y el bloqueo de los receptores adrenérgicos presinápticos, que disminuyen la actividad vasomotora central. En varios estudios y metaanálisis, los betabloqueadores no han logrado proporcionar beneficios como antihipertensivos en monoterapia de primera línea, en comparación con otras terapias ${ }^{23}$. Sólo muestran pequeñas reducciones en los eventos cardiovasculares y pocos efectos significativos sobre la mortalidad ${ }^{42}$.

Se deben usar en terapia de combinación, por lo general con diuréticos en pacientes de edad avanzada, que tienen afecciones cardiovasculares adicionales, tales como enfermedad cardíaca coronaria, particularmente en aquellos con antecedentes de infarto de miocardio, la insuficiencia cardiaca sistólica o arritmias $^{30}$.

La evidencia disponible no apoya su uso como fármacos de primera línea en el tratamiento de la hipertensión. Esta conclusión se basa en su efecto relativamente débil en la reducción de los ataques cerebrovasculares, y en la ausencia de efecto sobre las enfermedades cardiovasculares, en comparación con el placebo o ningún tratamiento. Más importante aún, se basa en la tendencia hacia peores resultados en comparación con los bloqueadores de canales de calcio, inhibidores del sistema renina-angiotensina y diuréticos tiazídicos. La mayoría (75\%) de la evidencia de estas conclusiones proviene de ensayos en los que el betabloqueante utilizado fue el atenolol ${ }^{42}$.

\section{Valoración de la necesidad, efectividad (objetivo terapéutico) y seguridad}

\section{Necesidad}

En todos los pacientes de edad avanzada con HTA, el tratamiento farmacológico antihipertensivo debe iniciarse de 
forma razonablemente rápida, con el fin de disminuir el efecto de los factores de riesgo cardiovascular. En este sentido, el tratamiento farmacológico antihipertensivo debe contemplarse como parte fundamental del manejo integral del paciente con HTA, cuyo objetivo final es reducir el riesgo cardiovascular. De acuerdo con la revisión realizada, hay coincidencia en que existen cinco clases principales de fármacos con los que se ha demostrado que reducir la PA conlleva una clara mejoría del pronóstico cardiovascular en los pacientes de edad avanzada: diuréticos, betabloqueadores, antagonistas del calcio, IECA y ARA-II.

De igual manera, existen unos umbrales que deben ser tenidos en cuenta para iniciar el tratamiento antihipertensivo. Por ejemplo, el JNC-8 propone los umbrales en las siguientes recomendaciones ${ }^{26}$ :

- En personas mayores de 60 años se debe iniciar tratamiento antihipertensivo a partir de cifras de PA mayores a $150 / 90 \mathrm{~mm} \mathrm{Hg}$, buscando una meta de PA menor a $150 / 90 \mathrm{~mm} \mathrm{Hg}$ (recomendación fuerte - grado A).

- No hay beneficio en tratar la PA de un paciente buscando una presión diastólica menor a $90 \mathrm{~mm} \mathrm{Hg}$. Sin embargo, si la presión diastólica es mayor a $90 \mathrm{~mm} \mathrm{Hg}$, el paciente sí requiere tratamiento antihipertensivo (para edades entre 30 a 59 años, recomendación fuerte - grado A. Para edades de 18 a 29 años, opinión de expertos - grado E).

- En la población general menor de 60 años, se debe iniciar tratamiento si la presión sistólica es mayor/igual a $140 \mathrm{~mm} \mathrm{Hg}$, buscando una meta de PAS menor a $140 \mathrm{~mm}$ $\mathrm{Hg}$ (opinión de expertos - grado $\mathrm{E}$ ).

- La población mayor de 18 años con enfermedad renal crónica (ERC) y PA mayor a $140 / 90 \mathrm{~mm} \mathrm{Hg}$ se beneficia al disminuir la presión a menos de $140 / 90 \mathrm{~mm} \mathrm{Hg}$ (opinión de expertos - grado $\mathrm{E}$ ).

- En la población mayor de 18 años con diabetes, se inicia tratamiento farmacológico si la PA es mayor a $140 / 90 \mathrm{~mm}$ $\mathrm{Hg}$, buscando una meta por debajo de 140/90 mm Hg (opinión de expertos - grado $\mathrm{E}$ ).

- En la población general (sin incluir la raza negra), incluidos pacientes con diabetes, el tratamiento antihipertensivo inicial debería agregar un diurético tipo tiazida, bloqueador de canal de calcio, IECA o ARA-II (recomendación moderada - grado $\mathrm{B}$ ).

- En la población general de raza negra, incluidos aquellos con diabetes, el tratamiento antihipertensivo inicial debería agregar un diurético tipo tiazida o bloqueador de los canales de calcio (para la población general afrodescendiente: recomendación moderada - grado B; para los pacientes afrodescendientes diabéticos: recomendación débil - grado C).

- En la población mayor de 18 años, con ERC e HTA, el tratamiento antihipertensivo inicial debería incluir un IECA o ARA-II para mejorar las consecuencias renales. Esto se aplica a todos los pacientes con ERC con HTA independiente de la raza o de si padece diabetes (recomendación moderada - grado B).

\section{Efectividad. Logro del objetivo terapéutico}

Para evaluar la efectividad del tratamiento antihipertensivo es importante tener presentes las cifras de presión arterial sistólica (PAS) y diastólica (PAD). De forma similar a lo esperado con cualquier otro grupo poblacional, en los pacientes de edad avanzada, una medición precisa de la PA es esencial para planificar la terapia. Es importante considerar la posibilidad de problemas particulares en la medición de la PA en estos pacientes, incluyendo hipotensión ortostática inducida por medicamentos, hipertensión de bata blanca y enfermedad aterosclerótica avanzada con arterias rígidas.

El control de la presión arterial en el hogar o la automonitorización en casa (AMC) y la monitorización ambulatoria de la presión arterial (MAPA), desempeñan un papel vital en el manejo del paciente con HTA. La MAPA proporciona la evaluación más precisa de la PA durante un período prolongado. Varios estudios recientes han demostrado la utilidad de la MAPA en la mejoría del valor predictivo de los valores de la PA y del control de la misma ${ }^{5,43,44}$. En la tabla 2 se evidencian los aspectos a tener en cuenta en la medición de la presión arterial.

En Colombia, acorde con la GPC de HTA, como recomendación fuerte a favor, durante el seguimiento de pacientes con HTA, debe preferirse la MAPA frente a la AMC, siempre que exista exceso de variabilidad en otras mediciones o respuestas insatisfactorias o inciertas en el cumplimiento de metas de tratamiento ${ }^{24}$.

Otro aspecto fundamental que contribuye al logro de los objetivos terapéuticos es la adherencia al tratamiento. Por ejemplo, el estudio de Sandhiya ${ }^{45}$, resalta la importancia de la educación a los pacientes de edad avanzada y a sus proveedores de atención en salud, sobre la importancia y el uso adecuado de los medicamentos, y de la relevancia de mejorar la adherencia para su bienestar, aspecto este último en el que se requiere mayor esfuerzo para mejorar el control de la presión arterial en dichos pacientes ${ }^{15}$.

Por otro lado, acorde con el estudio de Jacobs ${ }^{46}$, el déficit cognitivo en pacientes de edad avanzada afecta el grado de adherencia a la terapia farmacológica, así que debe ser valorado y mejorado como parte de un programa de atención farmacéutica, para otimizar la adherencia al tratamiento. Además, para mejorar la adherencia y el cumplimiento de metas como recomendación fuerte a favor, durante el tratamiento de los pacientes con HTA se recomienda ofrecer programas estructurados de seguimiento, liderados por equipos multidisciplinarios que impartan educación, motivación y soporte a cargo de personal entrenado ${ }^{22}$.

\section{Seguridad}

Debido a que la edad avanzada deteriora la función de los vasos sanguíneos y conduce a enfermedad cardiovascular, se requiere que la selección de los medicamentos para el tratamiento de la hipertensión arterial ofrezca las alternativas más seguras con base en las necesidades y características del paciente.

De acuerdo con el JNC-8, como opinión de expertos, cada estrategia debe ser ajustada según las circunstancias individuales, las preferencias del clínico y del paciente, y la tolerabilidad a los medicamentos. Además, en situaciones específicas, un medicamento antihipertensivo puede ser sustituido por otro si se percibe que hay efectos adversos ${ }^{26}$.

En general, los medicamentos antihipertensivos de primera línea, incluyendo diuréticos, IECA, ARA-II, 
Tabla 2 Método para la medición de la presión arterial

\begin{tabular}{|c|c|c|}
\hline \multicolumn{3}{|c|}{ Método para la medición de la presión arterial en la consulta } \\
\hline \multirow[t]{3}{*}{ Tiempos antes de la medición } & 1 hora & Evitar el café, los alimentos, el tabaquismo, los descongestionantes \\
\hline & 30 minutos & Evitar el ejercicio \\
\hline & 5 minutos & Sentado tranquilamente \\
\hline Preparación & & $\begin{array}{l}\text { El vaciado de la vejiga y del intestino y la eliminación de toda la } \\
\text { ropa que cubre el lugar de colocación del brazalete }\end{array}$ \\
\hline Medio ambiente & & Lugar tranquilo y cálido \\
\hline \multirow[t]{4}{*}{ Durante la medición } & $\begin{array}{l}\text { Posición del } \\
\text { cuerpo }\end{array}$ & $\begin{array}{l}\text { Sentado, espalda apoyada, sin cruzar las piernas, pies apoyados } \\
\text { sobre el piso, y relajado. }\end{array}$ \\
\hline & Brazo & Apoyado, usando el brazo con mayor valor (más cercano corazón) \\
\hline & Brazalete & A la altura del corazón, usando un tamaño adecuado \\
\hline & Medición & $\begin{array}{l}\text { Hacer dos mediciones, espaciadas } 1-2 \text { minutos de diferencia, y } \\
\text { realizar medición adicional si es necesario. Hacer medición de la } \\
\text { frecuencia cardíaca mediante la palpación de pulsos (por lo menos } \\
30 \text { segundos) después de la segunda medición. }\end{array}$ \\
\hline Después de la medición & & $\begin{array}{l}\text { Hacer lecturas de la presión arterial promedio, sin aproximarlas; } \\
\text { dejar registro. }\end{array}$ \\
\hline
\end{tabular}

Tomada y adaptada de: Chern-En Chiang, Tzung-Dau Wang, Kwo-Chang Ueng, et al. Life style modification for managing hypertension (S-ABCDE). 2015 Guidelines of the Taiwan Society of Cardiology and the Taiwan. Journal of the Chinese Medical Association. 2015;78:1e47.

bloqueadores de los canales del calcio y betabloqueadores, son bien tolerados por la mayoría de los pacientes de edad avanzada, en comparación con los más jóvenes. Sin embargo, los de edad avanzada tienen mayor riesgo de efectos adversos graves, incluida la interacción con otros medicamentos, relacionados con la polimedicación ${ }^{47}$. En la tabla 3 se detallan los problemas de seguridad más comunes con los diferentes grupos de antihipertensivos.

\section{Aspectos prácticos}

\section{Lo que el médico debe tener en cuenta}

Existen múltiples guías actualizadas de medicina basada en la evidencia, que sirven como referencia para la prescripción adecuada de medicamentos para el tratamiento de la hipertensión en pacientes de edad avanzada. Para esto se requiere que el clínico considere en todos los casos la razón beneficio/riesgo, para así mejorar la calidad de vida de este grupo de pacientes que va en aumento rápidamente ${ }^{23}$. Por ejemplo, los resultados del estudio SPRINT, demostraron tasas más bajas de eventos cardiovasculares y de mortalidad en pacientes de edad avanzada, con metas control de presión arterial sistólica de menos de $120 \mathrm{~mm} \mathrm{Hg}$. Sin embargo, la frecuencia de algunos eventos adversos (hipotensión, síncope, alteraciones electrolíticas y falla renal), fue más significativa que con pacientes con una meta de control de presión arterial sistólica de menos de $140 \mathrm{~mm} \mathrm{Hg}{ }^{31}$.

En la tabla 4 se comparan las metas de presión arterial de cuatro guías actualizadas, además de los medicamentos de primera línea para el tratamiento de la hipertensión en pacientes de edad avanzada. No obstante, estas directrices no son obligatorias ya que el tratamiento de la HTA debe ser individualizado, acorde con las características clínicas de cada paciente y, en última instancia, la decisión del médico sigue siendo de suma importancia en el manejo de la hipertensión.

\section{Lo que el farmacéutico debe tener en cuenta}

El acompañamiento por parte del farmacéutico en el tratamiento de los pacientes de edad avanzada, desempeña un papel relevante, principalmente en el seguimiento del tratamiento farmacológico, las actividades educativas que tiendan a mejorar los hábitos y estilos de vida, y el acompañamiento para el logro de la adherencia terapéutica. Además, acorde con la guía de práctica clínica de hipertensión en Colombia, como recomendación fuerte a favor, se establece que durante el tratamiento de los pacientes con HTA, para mejorar la adherencia al mismo y el cumplimiento de metas, se recomienda ofrecer programas estructurados de seguimiento, liderados por equipos multidisciplinarios que impartan educación, motivación y soporte a cargo de personal entrenado ${ }^{24}$.

\section{Discusión}

Los resultados de esta revisión estructurada permiten destacar cómo la hipertensión arterial es un factor de riesgo para la morbilidad y mortalidad cardiovascular, principalmente en los pacientes de edad avanzada. En esto coinciden numerosos estudios clínicos, los cuales no solo han demostrado que el tratamiento de la hipertensión en esta población contribuye a la disminución de ataque cerebrovascular, insuficiencia cardiaca, infarto de miocardio y mortalidad, sino que, además, han servido para la elaboración de diferentes guías de tratamiento de la hipertensión arterial en distintos países ${ }^{14,24,26-30,34,35}$. Todas estas guías se convierten en los documentos de referencia para cada uno de los países para los cuales están elaboradas.

Este trabajo recopila información que puede aportar al tratamiento farmacológico y no farmacológico de los pacientes de edad avanzada. Las guías existentes incluyen el tratamiento de pacientes de edad avanzada, ya que se sabe que este grupo poblacional va en crecimiento, 
Tabla 3 Principales reacciones adversas a medicamentos con antihipertensivos

\begin{tabular}{|c|c|c|c|c|}
\hline \multicolumn{5}{|c|}{ Principales reacciones adversas de los medicamentos antihipertensivos de primera línea en pacientes de edad avanzada } \\
\hline $\begin{array}{l}\text { Grupo } \\
\text { antihipertensivo }\end{array}$ & $\begin{array}{l}\text { Reacciones adversas } \\
\text { de mayor incidencia }\end{array}$ & $\begin{array}{l}\text { ¿Qué tan grave es } \\
\text { para el paciente? }\end{array}$ & $\begin{array}{l}\text { ¿Qué tan frecuente es } \\
\text { la RAM? }\end{array}$ & $\begin{array}{l}\text { ¿Cómo se minimizan } \\
\text { los efectos? }\end{array}$ \\
\hline $\begin{array}{l}\text { Inhibidores de la } \\
\text { enzima conversiva } \\
\text { de angiotensina }\end{array}$ & Tos seca $^{47}$ & Leve a moderado & $\begin{array}{l}\text { 0,5\% a } 2 \% \text { (captopril), } \\
1,3 \% \text { a } 2,2 \% \\
\text { (enalapril), } 2-10 \% \\
\text { (fosinopril), } 3,5 \% \\
\text { (lisinopril) }\end{array}$ & $\begin{array}{l}\text { Se resuelve } \\
\text { suspendiendo el } \\
\text { medicamento. } \\
\text { Alternativa un ARA II }\end{array}$ \\
\hline \multirow[t]{2}{*}{$\begin{array}{l}\text { Antagonistas de los } \\
\text { receptores AT1 de la } \\
\text { angiotensina II }\end{array}$} & Hipotensión & Leve & $\begin{array}{l}5,5 \% \text { a } 6,9 \% \\
\text { (valsartán) 18,8\% } \\
\text { (candesartán) }\end{array}$ & $\begin{array}{l}\text { Monitorización } \\
\text { periódica de la } \\
\text { presión arterial }\end{array}$ \\
\hline & Dolor de cabeza & Leve & $\begin{array}{l}\text { 1\% (valsartán, } \\
\text { candesartán), } 12.3 \% \\
\text { (irbesartán) }\end{array}$ & $\begin{array}{l}\text { Realizar seguimiento } \\
\text { de las RAM a los } \\
\text { pacientes }\end{array}$ \\
\hline \multirow[t]{5}{*}{ Diuréticos } & Hipomagnesemia & Leve & $\begin{array}{l}\text { No hay datos precisos } \\
\text { de incidencia. }\end{array}$ & $\begin{array}{l}\text { Disminuir dosis del } \\
\text { diurético }\end{array}$ \\
\hline & Hiponatremia ${ }^{47}$ & Leve & $\begin{array}{l}\text { Principalmente con } \\
\text { tiazidas. No hay datos } \\
\text { precisos de } \\
\text { incidencia. }\end{array}$ & $\begin{array}{l}\text { Disminuir dosis del } \\
\text { diurético; disminuir } \\
\text { el consumo de agua. }\end{array}$ \\
\hline & Hipopotasemia ${ }^{47}$ & Moderado & $\begin{array}{l}12,7 \% \text { (tiazidas) } 14,7 \% \\
\text { (bumetanida) }\end{array}$ & $\begin{array}{l}\text { Disminuir dosis del } \\
\text { diurético }\end{array}$ \\
\hline & Hiperglicemia ${ }^{17}$ & Leve & $\begin{array}{l}18,4 \% \text { (bumetanida y } \\
\text { diuréticos tiazídicos). }\end{array}$ & $\begin{array}{l}\text { Usar baja dosis de } \\
\text { diurético y combinar } \\
\text { con IECA. Ajustar } \\
\text { dosis de insulina. } \\
\text { Evitar combinación } \\
\text { con BB }\end{array}$ \\
\hline & $\begin{array}{l}\text { Hiperuricemia y } \\
\text { gota }^{48}\end{array}$ & Leve & $40 \%$ (furosemida) & $\begin{array}{l}\text { Suspender si el nivel } \\
\text { de potasio es }> \\
5,0 \mathrm{mEq} / \mathrm{L}, \mathrm{o} \\
\text { creatinina }>2,5 \\
\mathrm{mg} / \mathrm{dl} \text { en los } \\
\text { hombres, o }>2,0 \\
\mathrm{mg} / \mathrm{dl} \text { en mujeres. }\end{array}$ \\
\hline Calcio antagonistas & Estreñimiento ${ }^{47}$ & Leve & $\begin{array}{l}\text { 1,6\% (amlodipino), } \\
\text { menos del } 2 \%, \\
\text { (diltiazem), } 1 \% \\
\text { (nifedipino) }\end{array}$ & $\begin{array}{l}\text { Evitar administrar en } \\
\text { pacientes con } \\
\text { antecedentes de } \\
\text { estreñimiento. }\end{array}$ \\
\hline \multirow[t]{4}{*}{ Beta-bloquedores } & $\begin{array}{l}\text { Fatiga e intolerancia } \\
\text { al ejercicio }{ }^{47}\end{array}$ & Moderado & $\begin{array}{l}\text { Mayor al } 26 \% \\
\text { (atenolol), } 1 \% \text { a } 10 \% \\
\text { (metoprolol), } 24 \% \\
\text { (carvedilol) }\end{array}$ & $\begin{array}{l}\text { Realizar seguimiento } \\
\text { de las RAM a los } \\
\text { pacientes }\end{array}$ \\
\hline & Hipotensión & Moderado & $\begin{array}{l}4 \% \text { a } 25 \% \text { (atenolol), } \\
27,4 \% \text { (metoprolol), } \\
1,8 \% \text { a } 20,2 \% \\
\text { (carvedilol) }\end{array}$ & $\begin{array}{l}\text { Realizar seguimiento } \\
\text { de las RAM a los } \\
\text { pacientes }\end{array}$ \\
\hline & Extremidades frías & Moderado & $12 \%$ (atenolol) & $\begin{array}{l}\text { Realizar seguimiento } \\
\text { de las RAM a los } \\
\text { pacientes }\end{array}$ \\
\hline & Bradiarritmia & Moderado & $\begin{array}{l}3 \% \text { a } 18 \% \text { (atenolol), } \\
2 \% \text { a } 15,9 \% \\
\text { (metoprolol), } 3 \% \text { a } \\
10 \% \text { (carvedilol) }\end{array}$ & $\begin{array}{l}\text { Realizar seguimiento } \\
\text { de las RAM a los } \\
\text { pacientes }\end{array}$ \\
\hline
\end{tabular}


Tabla 4 Comparación de metas de presión arterial de cuatro guías clínicas de tratamiento

\begin{tabular}{|c|c|c|c|c|}
\hline \multicolumn{5}{|c|}{$\begin{array}{l}\text { Comparación de las guías: Guía de práctica clínica de hipertensión arterial en Colombia (GPC HTA Colombia 2013), Guías } \\
\text { europeas de hipertensión (ESH/ESC 2013), Octavo Comité Nacional Conjunto (JNC 8-2013) y Guía de la Sociedad de } \\
\text { Cardiología de Taiwán y la Sociedad de Hipertensión de Taiwán. } 2015 \text { (TSOC/THS 2015) }\end{array}$} \\
\hline & $\begin{array}{l}2013 \text { GPC HTA } \\
\text { Colombia }\end{array}$ & 2013 ESH/ESC & 2014 JNC-8 & 2015 TSOC/THS \\
\hline $\begin{array}{l}\text { Metas en la presión } \\
\text { arterial }\end{array}$ & $<140 / 90 \mathrm{mmHg}$ & $\begin{array}{l}\text { Universalmente } \\
<140 / 90 \mathrm{mmHg}\end{array}$ & $\begin{array}{l}<140 / 900 \\
<150 / 90 \mathrm{mmHg} \\
\text { para }>60)\end{array}$ & $\begin{array}{l}<140 / 90 \text { o } \\
<130 / 80 \mathrm{mmHg} \\
\text { para pacientes con } \\
\text { diabetes y } \\
\text { enfermedad } \\
\text { coronaria, o } \\
\text { enfermedad renal } \\
\text { crónica con } \\
\text { proteinuria. }\end{array}$ \\
\hline $\begin{array}{l}\text { Medicamentos de } \\
\text { primera línea }\end{array}$ & $\begin{array}{l}\text { ARA-II, IECA; BB; } \\
\text { BCC; diuréticos } \\
\text { tipo tiazídicos. }\end{array}$ & $\begin{array}{l}\text { Diurético tipo } \\
\text { tiazida, BB, BCC, } \\
\text { IECA o ARA-II }\end{array}$ & $\begin{array}{l}\text { Diurético tipo } \\
\text { tiazida, BCC, IECA } \\
\text { o ARA-II }\end{array}$ & $\begin{array}{l}\text { Diurético tipo } \\
\text { tiazida, BCC, IECA } \\
\text { o ARA-II }\end{array}$ \\
\hline Observaciones & $\begin{array}{l}\text { Aunque se } \\
\text { mantiene el uso } \\
\text { de } B B \text {, solo son } \\
\text { usados en } \\
\text { condiciones } \\
\text { específicas }\end{array}$ & $\begin{array}{l}\text { Mantiene el uso de } \\
\text { bloqueadores beta } \\
\text { como terapia en } \\
\text { condiciones } \\
\text { específicas }\end{array}$ & $\begin{array}{l}\text { Se excluyó el uso } \\
\text { de bloqueadores } \\
\text { beta como terapia } \\
\text { de primera línea } \\
\text { para hipertensión }\end{array}$ & $\begin{array}{l}\text { Se excluyó el uso } \\
\text { de bloqueadores } \\
\text { beta como terapia } \\
\text { de primera línea } \\
\text { para hipertensión }\end{array}$ \\
\hline
\end{tabular}

ARA-II: antagonistas de los receptores AT1 de la angiotensina II, IECA: inhibidores de enzima conversiva de angiotensina; BB: bloqueadores beta; BCC: bloqueadores de los canales de calcio.

tanto en países desarrollados como en vía de desarrollo. Sin embargo, son limitados los estudios clínicos que permiten generar directrices que faciliten el abordaje específico del problema de las personas mayores con múltiples enfermedades crónicas, con síndrome de fragilidad y también de pacientes de edad muy avanzada (mayores de $75 \mathrm{u}$ 80 años $)^{3,4,25}$.

Como aspecto diferenciador, el JNC-8 ${ }^{49}$ establece una meta de presión arterial menor a $150 / 90 \mathrm{~mm} \mathrm{Hg}$, en pacientes mayores de 60 años de edad, aspecto que difiere de las demás guías de tratamiento. Sin embargo, la guía taiwanesa de HTA, 2015 TSOC/THS establece la misma meta de presión arterial de menos de $150 / 90 \mathrm{~mm} \mathrm{Hg}$, pero sólo para pacientes mayores de 80 años (edad muy avanzada) ${ }^{30}$, lo cual coincide en cuanto a meta y edad relacionadas en el estudio HYVET $^{21}$.

Respecto al tratamiento farmacológico, en los diferentes artículos revisados concurre el que los medicamentos recomendados para iniciar la terapia, incluyen diuréticos tiazídicos, bloqueadores de los canales del calcio, IECA y ARA II, en pacientes diferentes a los de raza negra. Pese a ello, las guías europeas de hipertensión y de cardiología ${ }^{35}$, mantienen entre los medicamentos de primera línea de tratamiento a los betabloqueadores, mientras que la guía colombiana de hipertensión ${ }^{24}$, los mantiene solo para condiciones específicas de salud del paciente. De igual manera, hay coincidencia en que la individualización del tratamiento debe ser guiada por la presencia de factores de riesgo cardiovascular concomitantes.

En cuanto a las medidas no farmacológicas, en este trabajo se utilizó como referencia el sistema S-ABCDE que aparece descrito en la tabla 1, adaptado de la guía taiwanesa de hipertensión para la adopción de estilos de vida saludables, como parte fundamental en el tratamiento de la hipertensión. En este sentido, no se encuentran grandes cambios en las recomendaciones de otros trabajos. Por ejemplo, en el trabajo de Acelajado ${ }^{32}$ se plantea como en los pacientes de edad avanzada, la adopción de un estilo de vida saludable, que incluye dieta, actividad física y mantenimiento del peso corporal ideal, es la piedra angular de la gestión de la hipertensión, con beneficios que se extienden más allá de la reducción de la PA.

Esta revisión estructurada recopila aspectos relacionados con la necesidad, efectividad y seguridad del tratamiento farmacológico. Frente a esto se encuentran trabajos que mencionan aspectos relacionados con la seguridad de medicamentos específicos, pero no agrupan las recomendaciones importantes de los mismos, y mucho menos para pacientes de edad avanzada. De igual manera, cuando mencionan aspectos relacionados con la efectividad, se concuerdan en la forma de medir adecuadamente las cifras de presión arterial. En este sentido, esta revisión muestra un factor diferenciador que abarca aspectos claves para el tratamiento por parte del médico, y para el seguimiento que se les pueda realizar a estos pacientes de edad avanzada, por parte del farmacéutico clínico.

\section{Conclusión}

La revisión estructurada realizada permite identificar las características más importantes del tratamiento de la 
hipertensión arterial en pacientes de edad avanzada; adicionalmente, se lograron establecer recomendaciones de utilidad para el tratamiento de estos pacientes.

\section{Conflictos de interés}

Ninguno.

\section{Agradecimientos}

Al grupo de Promoción y Prevención Farmacéutica (PyPF) de la Universidad de Antioquia por su contribución a la revisión y redacción del artículo.

\section{Bibliografía}

1. OMS. Información general sobre la hipertensión en el mundo. Ginebra: Organización Mundial de la Salud; 2013.

2. Schäfer HH, De Villiers JN, Sudano I, Dischinger S, Theus GR, Zilla P, et al. Recommendations for the treatment of hypertension in the elderly and very elderly -a scotoma within international guidelines. Swiss Med Wkly. 2012;142: w13574.

3. Schwartz, Janice B. Primary prevention: Do the very elderly require a different approach? Trends Cardiovasc Med. 2015;25:228-39.

4. Gómez-Huelgas R, Giner-Galvañ V, Mostaza JM, Cuende JI, Miguel-Yanes JM, Rovira E, et al. Unanswered clinical questions in the management of cardiometabolic risk in the elderly: a statement of the Spanish society of internal medicine. BMC Cardiovascular Disorders. 2014;14:193.

5. Reddy AK, Jogendra MR, Rosendorff C. Blood pressure measurement in the geriatric population. Blood Press Monit. 2014;19:59-63.

6. Denker MG, Cohen DL. What is an appropriate blood pressure goal for the elderly: review of recent studies and practical recommendations? Clin Interv Aging. 2013;8:1505-17.

7. Pimenta E, Oparil S. Management of hypertension in the elderly. Nat Rev Cardiol. 2012;9:286-96.

8. Denker MG, Cohen DL. What is an appropriate blood pressure goal for the elderly: review of recent studies and practical recommendations? Clin Interv Aging. 2013;8:1505-17.

9. Rubio-Ruiz ME, Pérez-Torres I, Soto ME, Pastelín G, Guarner-Lans V. Aging in blood vessels, Medicinal agents FOR systemic arterial hypertension in the elderly. Ageing Res Rev. 2014;18:132-47. Epub 2014 Oct 13.

10. Cohen DL, Townsend RR. Update on pathophysiology and treatment of hypertension in the elderly. Curr Hypertens Rep. $2011 ; 13: 330-7$.

11. Lee HY, Oh BH. Aging and arterial stiffness. Circ J Off J Jpn Circ Soc. 2010;74:2257-62.

12. Beigel R, Dvir D, Arbel Y, Shechter A, Feinberg MS, Shechter M. Pulse pressure is a predictor of vascular endothelial function in middle-aged subjects with no apparent heart disease. Vasc Med Lond Engl. 2010;15:299-305.

13. Benetos A, Thomas F, Joly L, Blacher J, Pannier B, Labat C, et al. Pulse pressure amplification a mechanical biomarker of cardiovascular risk. J Am Coll Cardiol. 2010;55:1032-7.

14. O’Rourke MF, Adji A. Guidelines on guidelines: focus on isolated systolic hypertension in youth. J Hypertens. 2013;31:649-54.

15. Duprez D. Treatment of isolated systolic hypertension in the elderly. Expert Rev Cardiovasc Ther. 2012;10:1367-73.

16. Logan AG. Hypertension in aging patients. Expert Rev Cardiovasc Ther. 2011;9:113-20.
17. Sica DA, Carter B, Cushman W, Hamm L. Thiazide and loop diuretics. J Clin Hypertens. 2011;13.

18. Chobanian AV. Clinical practice. Isolated systolic hypertension in the elderly. N Engl J Med. 2007;357:789-96.

19. Spicuzza L, Gabutti A, Porta C, Montano N, Bernardi L. Yoga and chemoreflex response to hypoxia and hypercapnia. Lancet Lond Engl. 2000;356:1495-6.

20. Sangthong B, Ubolsakka-Jones C, Pachirat O, Jones DA. Breathing training for older patients with controlled isolated systolic hypertension. Med Sci Sports Exerc. 2016. In print.

21. Beckett NS, Peters R, Fletcher AE, Staessen JA, Liu L, Dumitrascu D, et al., for the HYVET Study Group. Treatment of hypertension in patients 80 years of age or older. N Engl J Med. 2008;358:1887-98.

22. Rosendorff C, Lackland DT, Allison M, Aronow WS, Black HR, Blumenthal RS, et al. Treatment of hypertension in patients with coronary artery disease. JACC. 2015;65.

23. Fleg JL, Aronow WS, Frishman WH. Cardiovascular drug therapy in the elderly: benefits and challenges. Nat Rev Cardiol. 2011;8:13-28.

24. Ministerio de Salud y Protección Social - Colciencias. Guía de práctica clínica Hipertensión arterial primaria (HTA). Bogotá; 2013.

25. Lisiak M, Uchmanowicz I, Wontor R. Frailty and quality of life in elderly patients with acute coronary syndrome. Clinical Interventions in Aging. 2016;11:553-62.

26. James PA, Oparil S, Carter BL, Cushman WC, DennisonHimmelfarb C, Handler J, et al. Evidence based-guideline for the management of high blood pressure in adults: Report from the panel members appointed to the Eighth Joint National Committee (JNC-8). JAMA. 2014, http://dx.doi.org/10.1001/jama.2013.284427. Publicación electrónica previa a la versión impresa. Disponible en http://jama.jamanetwork.com/journal.aspx

27. Zhang PY. Review of new hypertension guidelines. Eur Rev Med Pharmacol Sci. 2015;19:312-5.

28. Filipovský J, Widimský J, Špinar J. Summary of 2013 ESHESC Guidelines for the managetment of arterial hypertension. Coretvasa. 2014;56:e494-518.

29. Seedat YK, Rayner BL, Veriava Y. South African hypertension practice guideline 2014. Cardiovascular Journal of Africa. 2014;25, 6.

30. Chiang CE, Wang TD, Ueng CH, Lin TH, Yeh HI, Chen CY, et al. 2015 Guidelines of the Taiwan Society of Cardiology and the Taiwan. J Chinese Medical Association. 2015;78:1e47.

31. Wright JT Jr, Williamson JD, Whelton PK, Snyder JK, Sink KM, Rocco MV, et al., SPRINT Research Group. A randomized trial of intensive versus standard blood-pressure control. N Engl J Med. 2015;373:2103-16.

32. Acelajado MC, Oparil S. Hypertension in the Elderly. Clin Geriatr Med. 2009;25:391-412.

33. Appel LJ, On behalf of the American Society of Hypertension Writing Group. ASH Position Paper on dietary approaches to prevent and treat hypertension. J Clin Hypertens, Accessed June. 2009;18.

34. NICE. National Institute for Health and Clinical Excellence. Clinical guideline 127. Hipertensión. Manejo clínico de hipertensión primaria en adultos; 2015.

35. $2013 \mathrm{ESH} / \mathrm{ESC}$ guidelines for the management of arterial hypertension: the Task Force for the management of arterial hypertension of the European Society of Hypertension (ESH) and of the European Society of Cardiology (ESC). Eur Heart J. 2013;34:2159-219.

36. Wehling $M$. Morbus diureticus in the elderly: epidemic overuse of a widely applied group of drugs. J Am Med Dir Assoc. 2013;14:437-42.

37. Kaiser EA, Lotze U, Schäfer HH. Increasing complexity: which drug class to choose for treatment of 
hypertension in the elderly? Clin Interv Aging. 2014;9: 459-75.

38. Law MR, Morris JK, Wald NJ. Use of blood pressure lowering drugs in the prevention of cardiovascular disease: meta-analysis of 147 randomised trials in the context of expectations from prospective epidemiological studies. BMJ. 2009;338:b1665.

39. Rojas E, Velasco M, Bermúdez V, Israili Z, Bolli P. Targeting hypertension in patients with cardiorenal metabolic syndrome. Curr Hypertens Rep. 2012;14:397-402.

40. Pappoe LS, Winkelmayer WC. ACE inhibitor and angiotensin II type 1 receptor antagonist therapies in elderly patients with diabetes mellitus: are they underutilized? Drugs Aging. 2010;27:87-94.

41. Thomas GN, Chan P, Tomlinson B. The role of angiotensin II type 1 receptor antagonists in elderly patients with hypertension. Drugs Aging. 2006;23:131-55.

42. Wiysonge CS, Bradley H, Mayosi BM, Maroney R, Mbewu A, Opie LH, Volmink J. Beta-blockers for hypertension. Cochrane Database Syst Rev. 2007:CD002003.

43. Butt TF, Branch RL, Beesley L, Martin U. Managing hypertension in the very elderly: effect of adverse drug reactions (ADRs) on achieving targets. J Hum Hypertens. 2010;24:514-8.
44. Formosa V, Bellomo A, Iori A, Gianturco V, D’Ottavio E, Mancinella $M$, et al. Management of hypertension with fixed dose combinations of candesartan cilexetil and hydrochlorothiazide: patient perspectives and clinical utility. Vasc Health Risk Manag. 2009;5:1043-58. Epub 2009 Dec 29. Review.

45. Sandhiya S, Adithan C. Drug therapy in elderly. J Assoc Physicians India. 2008;56:525-31.

46. Jacobs Ú, De Castro MS, Fuchs FD, Ferreira MB. The influence of cognition, anxiety and psychiatric disorders over treatment adherence in uncontrolled hypertensive patients. PLoS One. 2011;6:e22925.

47. Aronow WS. Hypertension in the Elderly. Clin Geriatr Med. 2009;25:579-90.

48. Franse LV, Pahor M, Di Bari M, Somes GW, Cushman WC, Applegate WB. Hypokalemia associated with diuretic use and cardiovascular events in the Systolic Hypertension in the Elderly Program. Hypertension. 2000;35:1025-30.

49. 2014 evidence-based guideline for the management of high blood pressure in adults: report from the panel members appointed to the Eighth Joint National Committee (JNC-8). JAMA. 2014;311:507-20. 